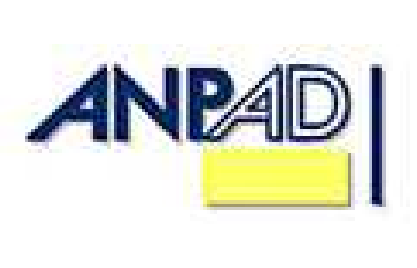

Disponível em

http://www.anpad.org.br/rac

RAC, Rio de Janeiro, v. 17, n. 1, art. 4,

pp. 64-82, Jan./Fev. 2013

$(\mathrm{cc}) \mathrm{EY}-\mathrm{NC}$

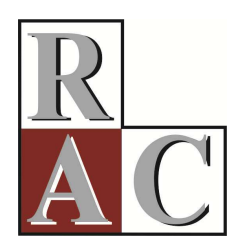

\title{
Desempenho Acadêmico e a Teoria do Prospecto: Estudo Empírico sobre o Comportamento Decisório
}

Academic Performance and Prospect Theory: an Empirical Study of Decision Behavior

Henrique Fonseca Genelhu Soares *

E-mail: henrique_7a@hotmail.com

Faculdades IBMEC

Rio de Janeiro, RJ, Brasil.

Claudio Henrique da Silveira Barbedo

E-mail: cbarbedo@ibmecrj.br

Faculdades IBMEC

Rio de Janeiro, RJ, Brasil.

* Endereço: Henrique Fonseca Genelhu Soares

Av. Presidente Wilson, 118, Rio de Janeiro/RJ, 20030-020.

Artigo recebido em 05.01.2012. Última versão recebida em 09.10.2012. Aprovado em 26.11.2012. 


\title{
Resumo
}

O presente trabalho tem por objetivo identificar a existência ou não de relação estatística entre a racionalidade das escolhas e o desempenho acadêmico dos discentes de Graduação de um Centro Universitário a partir do trabalho de Grinblatt, Keloharju e Linnainmaa (2011). Com base em questionários de coleta de dados e de regressões Logit, os resultados da pesquisa apontaram que a variável Desempenho Acadêmico (DA) apresenta relação direta com a racionalidade de escolhas e altera a ação dos vieses cognitivos sobre o processo decisório dos agentes. Nesse sentido, os discentes com desempenho acadêmico superior realizaram escolhas mais racionais que os estudantes com DA inferior, mostrando-se, dessa forma, menos susceptíveis à influência dos vieses cognitivos.

Palavras-chave: teoria dos prospectos; racionalidade das escolhas; desempenho acadêmico; vieses cognitivos; finanças comportamentais.

\begin{abstract}
This study aims to identify the existence of a statistical relationship between rational choice and academic performance of undergraduate university students, based on Grinblatt, Keloharju and Linnainmaa (2011). With the use of questionnaires to collect data and logit regressions, the results show that Academic Performance (AP) has a direct relationship with the rationality of choices and alters cognitive biases in decision making. In this sense, students with higher AP made more rational choices than students with lower AP, which means a lower susceptibility to cognitive biases.
\end{abstract}

Key words: prospect theory; rationality of choices; academic performance; cognitive biases; behavioral finance. 


\section{Introdução}

O mito de que investidores bem sucedidos no mercado de ações possuem uma inteligência diferenciada ou inata é uma questão que vem sendo alimentada pela mídia através de reportagens, artigos de revistas, livros especializados e biografias ${ }^{(1)}$. Entretanto, Kahneman e Tversky (1979) questionam a racionalidade nos investimentos, e sugerem que todos os investidores carregam características psicológicas e limitações que interferem nas suas decisões. No Brasil, por exemplo, Araújo e Silva (2007) verificam empiricamente que, como diversas decisões estão permeadas por incertezas e complexidades, os agentes de sua amostra não conseguem avaliar todas as variáveis contidas no processo. Cappellozza e Sanchez (2011) também evidenciam dissonâncias em decisões do setor de tecnologia.

O objetivo deste estudo é avaliar se o desempenho acadêmico pode ser um fator que contribui para a menor susceptibilidade à influência de vieses cognitivos. O estudo é baseado no trabalho de Grinblatt, Keloharju e Linnainmaa (2011). Para tal, o trabalho inicialmente identifica diferenças de comportamento entre o agente real e o agente modelado pelas finanças tradicionais, nos quesitos de aversão ao risco sobre ganhos e propensão ao risco sobre perdas, e tenta explicar a racionalidade das decisões através da variável Desempenho Acadêmico. Na parte inicial, o estudo replica a investigação empírica desenvolvida por Kahneman e Tversky (1979) com estudantes de graduação dos cursos de Administração e Saúde ${ }^{(2)}$ de uma instituição de ensino superior. A explicação da racionalidade via desempenho acadêmico é a principal contribuição deste trabalho.

O conceito de que os indivíduos são avessos ao risco sobre ganhos e propensos ao risco sobre perdas surgiu a partir da Teoria do Prospecto, com base no estudo seminal de dois psicólogos israelenses, Kahneman e Tversky. As conclusões dos autores sugeriram diferenças relevantes entre o agente real e o agente modelado pelas finanças tradicionais. Kahneman e Tversky (1981) apontam que o julgamento intuitivo, utilizado pelos indivíduos em tomadas de decisões financeiras, pode ser influenciado por heurísticas, ilusões e vieses cognitivos, acarretando decisões equivocadas e não baseadas na racionalidade.

Kimura, Basso e Krauter (2006) replicam, no Brasil, os experimentos de Kahneman e Tversky (1979), e os resultados mostram que se mantêm as evidências de diversos vieses de percepção em decisões, independentemente de aspectos relacionados com a liquidez do mercado e com a cultura ou a nacionalidade dos indivíduos. Silva, Lagioia, Maciel e Rodrigues (2009) também replicam o mesmo trabalho em um grupo de estudantes de faculdades no Brasil, e verificam que os resultados são muito similares aos da literatura, isto é, há a identificação dos vieses de julgamento, independente do semestre acadêmico no qual o aluno se encontra.

A contribuição deste trabalho é utilizar o resultado médio obtido por cada estudante no conjunto das disciplinas por ele cursadas, variável denominada desempenho acadêmico (DA), para investigar se estudantes com melhor desempenho acadêmico são mais racionais e menos susceptíveis à influência de vieses cognitivos do que os alunos com pior desempenho acadêmico. A base teórica é motivada pelos trabalhos seminais de Chevalier e Ellison (1999) e Gottesman e Morey (2006), que verificaram que o desempenho dos fundos de investimento é correlacionado com o resultado do gestor no exame SAT, GMAT ou com a sua classificação no programa de $\mathrm{MBA}^{(3)}$. Recentemente, Masood e Piranfar (2010) ratificaram esses resultados para o mercado turco, e Grinblatt et al. (2011) verificam que investidores com maior desempenho intelectual são menos sujeitos a vieses cognitivos. Dessa forma, a suposição em comum é que investidores com melhor desempenho acadêmico apresentam mais informação e, com isso, são menos propensos à tomada de decisão baseada em estruturas mentais.

As estruturas mentais formam uma importante categoria de ilusões cognitivas que afetam o processo de tomada de decisão dos agentes. Segundo Reed (1999), estruturas mentais são modelos de instrução, ou informais, capazes de facilitar a tomada de decisão e que podem ser influenciadas por várias características, como idade, desempenho acadêmico ou nível de renda. Mendes-da-Silva e Yu 
(2009), por exemplo, verificam que pessoas mais jovens têm menor senso de controle. As estruturas mentais discutidas no trabalho serão o Efeito Certeza e o Efeito Reflexo.

O Efeito Certeza foi detectado a partir de comparações que envolviam eventos considerados certos com eventos tidos como prováveis. Segundo Silva et al. (2009), os indivíduos tendem a conceder maior peso às possibilidades que têm alta probabilidade de acontecerem em detrimento aos resultados meramente prováveis, o que impulsiona a aversão ao risco nas escolhas que envolvem ganhos certos. O Efeito Reflexo foi identificado a partir dos resultados obtidos com vários testes que visavam definir as melhores opções entre as possibilidades de ganho ou entre diferentes possibilidades de perda. Kahneman e Tversky (1979) argumentam que os indivíduos tendem a ser avessos ao risco no domínio dos ganhos e propensos ao risco em situações que envolvam perdas.

Os resultados desta pesquisa sugerem que os discentes com DA superior realizam escolhas mais racionais que os estudantes com DA inferior. Neste sentido, conforme será destacado posteriormente, o DA apresenta relação direta com a racionalidade de escolhas e, aparentemente, altera a ação dos vieses cognitivos sobre o processo decisório dos agentes. Além disso, os resultados sugerem que os indivíduos são avessos ao risco no domínio dos ganhos e propensos ao risco no domínio das perdas.

Este trabalho está organizado da seguinte maneira: a segunda seção apresenta a amostra e metodologia do trabalho; a terceira seção comenta os resultados; e a quarta seção ilustra as conclusões do estudo.

\section{Metodologia e Amostra}

As hipóteses investigadas neste trabalho visam inferir a aversão ao risco no domínio dos ganhos e das perdas, e a relação entre racionalidade e desempenho acadêmico. Assim, as seguintes hipóteses serão avaliadas:

1. Hipótese 1:

$\mathbf{H}_{\mathbf{0}}$ - Os indivíduos são avessos ao risco no domínio dos ganhos, ou seja, as alternativas que apresentam ganhos certos são preferidas em relação aos prospectos que envolvem probabilidades incertas.

$\mathbf{H}_{1}$ - Os indivíduos não são avessos ao risco no domínio dos ganhos, ou seja, as alternativas que apresentam ganhos certos são preteridas (ou indiferentes) em relação aos prospectos que envolvem probabilidades incertas.

2. Hipótese 2:

$\mathbf{H}_{0}$ - Os indivíduos são propensos ao risco no domínio das perdas, ou seja, os prospectos que apresentam perdas certas são menos preferidos que as alternativas que envolvem probabilidades incertas.

$\mathbf{H}_{1}$ - Os indivíduos não são propensos ao risco no domínio das perdas, ou seja, os prospectos que apresentam perdas certas são preferidos (ou indiferentes) em relação às alternativas que envolvem probabilidades incertas.

3. Hipótese 3:

$\mathbf{H}_{0}$ - Os estudantes com melhor desempenho acadêmico são mais racionais e menos susceptíveis à influência de vieses cognitivos que os alunos com pior desempenho escolar. 
$\mathbf{H}_{1}$ - Os estudantes com melhor desempenho acadêmico são menos racionais (ou igualmente racionais) e mais susceptíveis (ou igualmente susceptíveis) à influência de vieses cognitivos que os alunos com pior desempenho escolar.

No contexto do estudo, a racionalidade embutida na hipótese 3 será avaliada por meio de conjuntos de escolhas racionais, isto é, através de escolhas simultâneas que indicam a racionalidade e a padronização da forma como os indivíduos interpretam os problemas. Assim, torna-se desnecessário estimar a função utilidade de cada respondente, dado que a racionalidade implica a coerência de escolhas e não a importância individual concedida a cada prospecto. Já os vieses cognitivos serão avaliados através da influência no processo decisório dos efeitos representados no questionário: o efeito certeza e o efeito reflexo.

\section{Método de coleta e análise de dados}

Para o alcance dos objetivos propostos, a coleta de dados ocorre através da utilização de questionário (Apêndice). O questionário é composto por questões fechadas, com base na metodologia proposta por Kahneman e Tversky (1979). As alterações realizadas para adequação à realidade brasileira são adaptadas por Kimura et al. (2006) e Silva et al. (2009). O questionário é composto por duas partes. A primeira visa coletar as características dos indivíduos, como sexo e idade, curso de graduação e período. A segunda parte consiste de problemas nos quais os indivíduos são submetidos a tomar decisões, tendo por base alternativas hipotéticas de certeza ou de incerteza.

Os problemas apresentam duas opções de resposta (A e B), em que a escolha por uma delas representa um cenário de ganho ou de perda. $\mathrm{O}$ questionário possibilita a análise da existência dos efeitos certeza e reflexo. O efeito certeza é verificado através das questões 1 a 8 e o efeito reflexo pelos problemas 9 a 12. O problema 13 visa avaliar a influência da estruturação dos prospectos e o efeito isolamento. Seguindo a sugestão de Kimura et al. (2006), os prospectos serão apresentados em ordem distinta para diferentes participantes, de modo a minimizar os problemas relativos a potenciais efeitos da ordem das alternativas sobre os respondentes.

A principal variável da pesquisa a ser levantada, o desempenho acadêmico (DA), será mensurada por meio de uma média aritmética simples das notas de cada aluno em todas as disciplinas por eles cursadas nos dois últimos períodos ${ }^{(4)}$. Dessa forma, os alunos serão classificados dentro de três categorias, numa escala que varia de 0 a 100: (a) alunos com baixo desempenho acadêmico, ou seja, com DA $\leq 75$; (b) alunos com desempenho acadêmico intermediário, isto é, com $75<\mathrm{DA} \leq 85$; (c) estudantes com ótimo desempenho acadêmico, ou seja, com $85<\mathrm{DA} \leq 100^{(5)}$.

\section{Amostra}

A população alvo da pesquisa é composta por estudantes dos cursos de graduação em Administração e Saúde ${ }^{(6)}$ de uma Instituição de Ensino Superior Privada sem fins lucrativos. A escolha para compor a amostra foi feita de forma aleatória, por sorteio, em um mesmo período acadêmico. A participação dos alunos foi voluntária, e a aplicação do questionário ocorreu mediante presença em sala de aula. Do total de 250 questionários aplicados (metade em cada curso), 207 foram coletados, sendo 88 respondidos pelos alunos do curso de Administração (42,51\%) e 119 pelos de Saúde (57,49\%). No curso de Administração, 35, ou 39,77\%, são homens, enquanto que, na Saúde, apenas 16 participantes são do sexo masculino (13,45\%). Em média, os estudantes de Administração $(22,52$ anos) são ligeiramente mais velhos do que os de Saúde (21,16 anos). Além disso, o desvio-padrão inferior da Idade para os acadêmicos de Administração $(4,53)$ indica que as divergências de faixa etária entre este grupo são menores do que para os estudantes de Saúde $(4,86)$. No geral, a idade média dos respondentes foi de 21,74 anos, sendo 44 anos o valor máximo e 18 anos o valor mínimo encontrados na amostra.

Cerca de 55\% dos estudantes de Administração enquadraram-se com desempenho acadêmico intermediário. A minoria dos respondentes de Administração, ou 19 alunos, foram classificados com 
desempenho acadêmico superior. No curso da área de Saúde, a parcela majoritária dos estudantes $(51,26 \%)$ registrou desempenho acadêmico inferior. A minoria, ou 16,8\%, por sua vez, foi classificada com desempenho acadêmico superior (DA > 85), atingindo uma média de notas de 87,58 pontos.

Em termos comparativos, os alunos de Administração posicionaram-se em um nível de desempenho acadêmico superior aos estudantes de Saúde, isto é, com média de notas de 79,2 contra 74,25 pontos. Há, ainda, menor disparidade de notas entre os participantes do curso de Administração, refletido através do máximo e mínimo. Com isso, a fim de evitarmos julgamentos subjetivos de desempenho escolar, todas as comparações serão realizadas apenas considerando o próprio curso de cada aluno. A Tabela 1 apresenta a composição do desempenho acadêmico dos alunos.

Tabela 1

Composição do Desempenho Acadêmico dos Alunos de Administração e Saúde

\begin{tabular}{lcccccccc}
\hline & \multicolumn{1}{l}{ Saúde } & \multicolumn{5}{c}{ Administração } \\
\cline { 2 - 9 } Desempenho Acadêmico & $\mathbf{N}^{\circ}$ & Mediana & Máximo & Mínimo & $\mathbf{N}^{\circ}$ & Mediana & Máximo & Mínimo \\
\hline Inferior & 61 & 61,9 & 74,3 & 35,25 & 21 & 69,55 & 75 & 56,5 \\
\hline Intermediário & 38 & 78,7 & 84,5 & 75,2 & 48 & 79,25 & 84,8 & 76 \\
\hline Superior & 20 & 87,2 & 91,8 & 85,1 & 19 & 86,1 & 88,8 & 85,1 \\
\hline Geral & 119 & 74,25 & 91,8 & 35,25 & 88 & 79,2 & 88,8 & 56,5 \\
\hline
\end{tabular}

\section{Resultados}

\section{Avaliação dos resultados por curso}

O viés do efeito certeza faz com que os agentes abandonem escolhas com utilidade esperada (resultados ponderados pela sua respectiva probabilidade de ocorrência) maior, em favor das opções que sinalizam proximidade para com os ganhos. Esse efeito é ilustrado pelos problemas 1 a 6 do questionário. As respostas dos alunos de Administração e Saúde são comparadas com as obtidas nos trabalhos de Kahneman e Tversky (1979) e Kimura et al. (2006). A pesquisa efetuada no Brasil é utilizada como parâmetro de comparação, pois sua amostra é composta por estudantes brasileiros. Assim, eliminam-se possíveis divergências culturais que envolvam o processo de tomada de decisão.

A Tabela 2 apresenta as respostas das três pesquisas para os problemas 1 a 8 . O estudo de Kahneman e Tversky (1979) foi referenciado como K\&T, enquanto o trabalho de Kimura et al. (2006) foi renomeado como Brasil. Da presente pesquisa, as opções escolhidas pelos alunos de Administração foram referidas como Adm e dos alunos de Saúde como Saúde. Ao lado das classificações encontra-se, em parênteses, a amostra respondente de cada problema. 
Tabela 2

Avaliação do Efeito Certeza

\begin{tabular}{cccccc}
\hline Problema & Prospecto & Adm (88) & Saúde (119) & K\&T (72) & Brasil (98) \\
\hline $\mathbf{1}$ & A & $39,70 \%$ & $23,50 \%$ & $18 \%$ & $30 \%$ \\
& B & $60,30 \%$ & $76,50 \%$ & $82 \%$ & $70 \%$ \\
\hline $\mathbf{2}$ & A & $57,90 \%$ & $24,30 \%$ & $83 \%$ & $52 \%$ \\
& B & $42,10 \%$ & $75,70 \%$ & $17 \%$ & $48 \%$ \\
\hline $\mathbf{3}$ & A & $31,80 \%$ & $20,20 \%$ & $20 \%$ & $29 \%$ \\
& B & $68,20 \%$ & $79,80 \%$ & $80 \%$ & $71 \%$ \\
\hline $\mathbf{4}$ & A & $44,30 \%$ & $46,20 \%$ & $65 \%$ & $57 \%$ \\
& B & $55,70 \%$ & $53,80 \%$ & $35 \%$ & $43 \%$ \\
\hline $\mathbf{5}$ & A & $17 \%$ & $15,10 \%$ & $22 \%$ & $20 \%$ \\
& B & $83 \%$ & Mendes $84,90 \%$ & $78 \%$ & $80 \%$ \\
\hline $\mathbf{6}$ & A & $40,20 \%$ & $21 \%$ & $67 \%$ & $49 \%$ \\
& B & $59,80 \%$ & $79 \%$ & $33 \%$ & $51 \%$ \\
\hline $\mathbf{7}$ & A & $22,70 \%$ & $20,20 \%$ & $14 \%$ & $23 \%$ \\
& B & $77,30 \%$ & $79,80 \%$ & $86 \%$ & $77 \%$ \\
\hline $\mathbf{8}$ & A & $55 \%$ & $43,70 \%$ & $73 \%$ & $72 \%$ \\
& B & $45 \%$ & $56,30 \%$ & $27 \%$ & $28 \%$ \\
\hline
\end{tabular}

A opção de 60,3\% dos alunos de Administração e de 76,5\% dos acadêmicos de Saúde pela alternativa B da questão 1 indica que os respondentes, conforme as pesquisas de Kahneman e Tversky (1979) e Kimura et al. (2006), são influenciados pelo efeito certeza. Nesse sentido, assumido que $\mathrm{U}(0)=0$, ou seja, que a utilidade de zero é igual a zero, tem-se que a maioria dos agentes definiram que $\mathrm{U}(2400)>0,33 \mathrm{U}(2500)+0,66 \mathrm{U}(2400)$ ou que $0,34 \mathrm{U}(2400)>0,33 \mathrm{U}(2500)$.

O problema 2, por sua vez, indica divergências de escolhas entre os acadêmicos de Administração e Saúde. Os alunos de Administração, em sua maioria, optam pela alternativa A, assim como nas pesquisas utilizadas como parâmetro. Dessa forma, contrariam a Teoria da Utilidade Esperada, pois definem exatamente o oposto do problema 1, ou seja, que 0,33U(2500) >0,34U(2400). Segundo Kimura et al. (2006), esse comportamento sugere inconsistência dos agentes, já que estes alteram sua ordem de preferências. Em contrapartida, $75,7 \%$ dos acadêmicos de Saúde preferem a alternativa $\mathrm{B}$, que implica que $0,34 \mathrm{U}(2400)>0,33 \mathrm{U}(2500)$. Assim, este grupo não demonstra a priori inconsistência de escolhas, dado que mantém suas preferências nos dois problemas, ainda que sejam as alternativas com menor utilidade esperada.

O problema 3 apresenta outro processo de escolha em que a influência do efeito certeza é notada. Os estudantes de Administração e Saúde optaram pela alternativa B, respectivamente, em $68,2 \%$ e $79,8 \%$ dos casos, embora a utilidade esperada da alternativa menos preferida (\$3200) exceda a da opção B (\$3000). No problema 4, a opção majoritária reflete, novamente, a alternativa com menor utilidade esperada. $\mathrm{O}$ problema 5 tenta inferir o efeito certeza em situações que não envolvam valores monetários. Em sintonia com as pesquisas de Kahneman e Tversky (1979) e Kimura et al. (2006), $83 \%$ e $84,9 \%$, respectivamente, dos alunos de Administração e Saúde preferiram a alternativa B, que assegura uma viagem de uma semana para a Inglaterra. Ainda que o prospecto A seja mais generoso (viagem de três semanas para Inglaterra, França e Itália), a incerteza embutida nessa opção torna o outro prospecto mais atraente. Na questão 6 , assim como na 4, os resultados indicam que os alunos podem ter sido influenciados pela probabilidade de ganho superior presente no prospecto $B$.

No problema 7, a ampla escolha de B indica que os estudantes entendem que 0,9U(3000) > $0,45 \mathrm{U}(6000)$, ainda que as utilidades esperadas de ambos os prospectos sejam iguais. Em situações 
que envolvam probabilidades ínfimas de ganho, questão 8, Kahneman e Tversky (1979) defendem que os agentes preferem os prospectos que fornecem ganhos superiores, ao invés de avaliarem as probabilidades. Nesse sentido, apenas os estudantes de Administração manifestaram suas escolhas conforme o preconizado pelos autores.

Em síntese, os problemas 1 a 8 revelam que os discentes de Administração e Saúde geralmente seguiram o padrão das pesquisas anteriores. Os resultados demonstraram que os respondentes são sensíveis ao efeito certeza, e corroboraram com a não rejeição de $\mathrm{H}_{0}$ da hipótese 1 , isto é, os indivíduos são avessos ao risco no domínio dos ganhos, as alternativas que apresentam ganhos certos são preferidas em relação às alternativas que envolvem probabilidades incertas.

Os problemas de 9 a 12 ilustram a influência do comportamento propenso ao risco dos indivíduos perante situações de perdas certas. A propensão ao risco dos indivíduos perante tais situações se confirma na questão 9 (vide Tabela 3), à medida que $78,4 \%$ e $72,3 \%$ dos respondentes de Administração e Saúde, respectivamente, optam pelo prospecto em que existe uma probabilidade minoritária de evitar a perda, mesmo que, caso ocorra, esta seja mais expressiva (-\$4000). Tal padrão de comportamento, constatado nas demais pesquisas, revela que os agentes, em média, possuem aversão às perdas, mas não aversão ao risco, como prevê o viés cognitivo efeito reflexo.

Tabela 3

Avaliação do Efeito Reflexo

\begin{tabular}{cccccc}
\hline Problema & Prospecto & Adm (88) & Saúde (119) & K\&T (95) & Brasil (97) \\
\hline $\mathbf{9}$ & A & $78,40 \%$ & $72,30 \%$ & $92 \%$ & $82 \%$ \\
& B & $21,60 \%$ & $27,70 \%$ & $8 \%$ & $18 \%$ \\
\hline \multirow{2}{*}{$\mathbf{1 0}$} & A & $52,20 \%$ & $52,10 \%$ & $42 \%$ & $37 \%$ \\
& B & $47,80 \%$ & $47,90 \%$ & $58 \%$ & $63 \%$ \\
\hline \multirow{2}{*}{11} & A & $70,50 \%$ & $55,50 \%$ & $92 \%$ & $75 \%$ \\
& B & $29,50 \%$ & $44,50 \%$ & $8 \%$ & $25 \%$ \\
\hline \multirow{2}{*}{$\mathbf{1 2}$} & A & $50 \%$ & $46,20 \%$ & $30 \%$ & $50 \%$ \\
& B & $50 \%$ & $53,80 \%$ & $70 \%$ & $50 \%$ \\
\hline
\end{tabular}

Outra forma de avaliar a aversão ou propensão ao risco é verificar que os problemas 3 e 9 são simétricos, porém o primeiro contempla ganhos. Entretanto, enquanto no problema 3, a escolha majoritária é pelo prospecto seguro, no problema 9, é pelo risco. Tal alteração de comportamento reflete, conforme assinalado, que os indivíduos são avessos ao risco no domínio dos ganhos, mas propensos ao risco no domínio das perdas. Da mesma forma que as questões 3 e 9 , os problemas 4 e 10 são relacionados e induzem à mesma resposta.

O problema 11 ressalta a preferência dos discentes de Administração e Saúde, assim como dos participantes das demais pesquisas, pelo prospecto A. O objetivo demonstrado perante situações desse tipo é minimizar as chances de perda, ainda que os valores possivelmente perdidos sejam maiores. Contrariamente ao assinalado no problema 11, parcela majoritária dos participantes sinalizou preferência pelo prospecto $\mathrm{B}$ do problema 7 , indicando a dualidade de comportamento dos agentes em face de cenários que englobam ganhos ou perdas. O problema 12, opção pela perda menor, ainda que esta escolha acarrete a duplicação da probabilidade envolvida, reforça o viés estudado.

Os resultados obtidos corroboraram com a não rejeição de $\mathrm{H}_{0}$ da hipótese 2, isto é, os indivíduos são propensos ao risco no domínio das perdas, ou seja, os prospectos que apresentam perdas certas são menos preferidos que as alternativas que envolvem probabilidades incertas. Tal resultado ratifica Kahneman e Tversky (1979) e Kimura et al. (2006) e ainda está em conformidade com os obtidos pelos autores Rogers, Securato e Ribeiro (2007), e Cruz, Kimura e Krauter (2003) ${ }^{(7)}$. 


\section{Resultados com desempenho acadêmico}

A partir da suposição de que alunos que compartilham o mesmo nível de desempenho acadêmico tendem a se comportar de forma semelhante em processo de decisão, avaliam-se os resultados dos questionários à luz das classificações definidas para o desempenho acadêmico. A Tabela 4 apresenta os resultados para os problemas 1 a 8 , que identificam a influência do efeito certeza no processo de tomada de decisão dos indivíduos.

Tabela 4

Avaliação do Efeito Certeza à Luz do Desempenho Acadêmico

\begin{tabular}{ccccc}
\hline Problema & Prospecto & DA Inferior & DA Intermediário & DA Superior \\
\hline $\mathbf{1}$ & A & $27,85 \%$ & $34,52 \%$ & $26,32 \%$ \\
& B & $72,15 \%$ & $65,48 \%$ & $73,68 \%$ \\
\hline $\mathbf{2}$ & A & $27,85 \%$ & $29,76 \%$ & $44,74 \%$ \\
& B & $72,15 \%$ & $70,24 \%$ & $55,26 \%$ \\
\hline $\mathbf{3}$ & A & $30,38 \%$ & $25 \%$ & $15,79 \%$ \\
& B & $69,62 \%$ & $75 \%$ & $84,21 \%$ \\
\hline $\mathbf{4}$ & A & $49,37 \%$ & $46,43 \%$ & $36,84 \%$ \\
& B & $50,63 \%$ & $53,57 \%$ & $63,16 \%$ \\
\hline $\mathbf{5}$ & A & $19 \%$ & $11,90 \%$ & $18,42 \%$ \\
& B & $81 \%$ & $88,10 \%$ & $81,58 \%$ \\
\hline $\mathbf{6}$ & A & $20,25 \%$ & $33,20 \%$ & $34,21 \%$ \\
& B & $79,75 \%$ & $66,80 \%$ & $65,79 \%$ \\
\hline $\mathbf{7}$ & A & $25,32 \%$ & $15,50 \%$ & $23,68 \%$ \\
& B & $74,68 \%$ & $84,50 \%$ & $76,32 \%$ \\
\hline $\mathbf{8}$ & A & $41,77 \%$ & $50 \%$ & $55,26 \%$ \\
& B & $58,23 \%$ & $50 \%$ & $44,74 \%$ \\
\hline
\end{tabular}

Os resultados do teste qui-quadrado, aplicado para determinar a significância das diferenças encontradas entre as proporções, sugerem para o problema 1 que a preferência pela certeza independe do desempenho acadêmico. Para um nível de significância de 5\%, encontramos um p-valor de 0,0616\%, o que significa a rejeição da hipótese nula de que não há diferença entre os valores observados. Nesse problema, cerca de $72,15 \%$ e 73,68\% dos estudantes com desempenho acadêmico inferior e superior, respectivamente, optaram pelo prospecto B, isto é, aquele que garante o ganho certo de $\$ 2400$. No problema 2, apenas $55,26 \%$ dos alunos com desempenho acadêmico superior definiram pelo prospecto $\mathrm{B}$ (o prospecto A apresenta maior utilidade esperada).

Em consonância com o problema 1, os resultados da questão 3 ilustram a preferência dos respondentes pelos prospectos que envolvem ganhos certos, independente dos resultados escolares. Nos problemas 4, 5, 6 e 7, o desempenho acadêmico não representou resposta diferenciada. No problema 8, somente os discentes com DA superior corroboraram com a argumentação de Kahneman, com 55,26\% optando pela alternativa A. Parcela majoritária dos estudantes com DA inferior $(58,23 \%)$ escolheram o prospecto $\mathrm{B}$, enquanto os alunos com DA intermediário posicionaram-se indiferentes quanto às opções disponíveis.

A Tabela 5 apresenta os resultados para os problemas 9 a 12, os quais avaliam os impactos do efeito reflexo no comportamento dos indivíduos. 
Tabela 5

Avaliação do Efeito Reflexo à Luz do Desempenho Acadêmico

\begin{tabular}{ccccc}
\hline Problema & Prospecto & DA Inferior & DA Intermediário & DA Superior \\
\hline \multirow{2}{*}{$\mathbf{9}$} & A & $70,90 \%$ & $80,95 \%$ & $71,05 \%$ \\
& B & $29,10 \%$ & $19,05 \%$ & $28,95 \%$ \\
\hline \multirow{2}{*}{$\mathbf{1 0}$} & A & $46,84 \%$ & $58,33 \%$ & $44,74 \%$ \\
& B & $53,16 \%$ & $41,67 \%$ & $55,26 \%$ \\
\hline \multirow{2}{*}{$\mathbf{1 1}$} & A & $50,63 \%$ & $69,05 \%$ & $71,05 \%$ \\
& B & $49,37 \%$ & $30,95 \%$ & $28,95 \%$ \\
\hline \multirow{2}{*}{$\mathbf{1 2}$} & A & $39,24 \%$ & $50 \%$ & $55,26 \%$ \\
& B & $60,76 \%$ & $50 \%$ & $44,74 \%$ \\
\hline
\end{tabular}

Os resultados obtidos no problema 9, com mais de $70 \%$ de todos os grupos apontando sua preferência pelo prospecto $\mathrm{A}$, indicam que os agentes participantes da pesquisa, independentemente do desempenho acadêmico auferido, são avessos às perdas, mas não ao risco, conforme prevê o efeito reflexo. No problema 10, a maioria dos discentes com desempenho acadêmico inferior $(53,16 \%)$ e superior $(55,26 \%)$ agiu conforme postula a Teoria da Utilidade Esperada, ao contrário dos estudantes com DA intermediário, que, em sua maioria, optaram pela alternativa A. Os problemas 11 e 12, apesar dos resultados mais favorecidos para os estudantes de DA superior, também indicam fortes indícios da presença do efeito reflexo no processo de tomada de decisão.

A estratificação da amostra por desempenho acadêmico, resultando em três classificações conforme as notas, sinalizou, a priori, que a influência dos vieses cognitivos é pouco dependente do nível de desempenho acadêmico. Entretanto, para que a avaliação da hipótese 3 seja precisa, opta-se por regressões com todas as informações disponíveis no questionário.

\section{Análise estatística dos dados}

A mensuração da racionalidade dos indivíduos é dada pela coerência de escolhas em cada conjunto de problemas apontados como complementares ou simétricos. Os problemas 1 e 2; 3 e 9; 5 e $6 ; 7$ e $11 ; 8$ e 12; 4 e 13 e 4 e 10 sugerem que a racionalidade é medida através das respostas em alternativas simultâneas (AA ou BB). Para a identificação de significância estatística entre a racionalidade das escolhas e o desempenho acadêmico, serão rodadas regressões $\operatorname{Logit}^{\left({ }^{(8)}\right.}$, sendo uma regressão geral para cada conjunto definido.

\section{Identificação do modelo}

A identificação inicial do modelo englobou, além da variável dependente RACIONAL, a variável DA (desempenho acadêmico), contínua em uma escala de 0 a 100 pontos, a variável dummy sexo e a variável idade.

As regressões iniciais para cada conjunto de problemas foram estimadas por curso e sinalizou a não significância das variáveis Sexo e Idade ${ }^{(9)}$. Entretanto, a variável desempenho acadêmico (DA) mostrou-se estatisticamente significante na maioria das vezes. Assim, com o objetivo de captar esse efeito, o modelo final selecionado englobará esta variável e o curso, que será dada por 1 para Administração e 0 para Saúde, em que P é a probabilidade do indivíduo ter optado por uma escolha racional. A composição do modelo geral será conforme a Equação (1).

$$
\ln \left(\frac{P}{1-P}\right)=\alpha_{0}+\beta_{1} D A_{i}+\beta_{2} \operatorname{Curso}_{i}+\varepsilon_{i}
$$


Os resultados da estimação para o conjunto 1 (problemas 1 e 2) são apresentados na Tabela 6. Nesse caso, o viés verificado é que as preferências por prospectos dependem não somente da utilidade atribuída aos resultados em si, mas também do nível de certeza do resultado. Variável dependente: RACIONAL.

Tabela 6

Modelo Geral para o Conjunto 1

\begin{tabular}{ccccc}
\hline Variáveis & Coeficiente & Erro Padrão & Estatística t & P-valor \\
\hline C & -2.415913 & 1.029455 & -2.346789 & 0.0189 \\
DA & 0.0369 & 0.014130 & 2.611459 & 0.0090 \\
Curso & 0.173875 & 0.314053 & 0.553650 & 0.5798 \\
& & McFadden $\mathrm{R}^{2}=0,0341$ & \\
\hline
\end{tabular}

A dummy de curso mostrou-se não significativa sob o ponto de vista estatístico, indicando que o curso realizado pelo estudante não influencia na sua tomada de decisões. Entretanto, o coeficiente positivo e significativo da variável DA aponta, nesse caso, para a existência de relação direta entre o desempenho acadêmico e a racionalidade das escolhas, isto é, quanto maior a média de notas auferida pelos estudantes, tanto maior será sua probabilidade de optar pelas alternativas AA ou BB. De qualquer forma, o percentual de oscilação da variável dependente captada pelas independentes $(3,41 \%)$ é pequeno, sugerindo que outras variáveis podem ser incluídas no modelo, visando elevar sua capacidade explicativa.

A Tabela 7 expõe os resultados do modelo geral para o conjunto 2, composto pelos problemas 3 e 9. Nesse caso, o viés é verificado pelo comportamento de aversão ao risco no domínio dos ganhos e de propensão ao risco no domínio das perdas.

Tabela 7

Modelo Geral para o Conjunto 2

\begin{tabular}{ccccc}
\hline & \multicolumn{3}{c}{ Variável dependente: RACIONAL } \\
Variáveis & Coeficiente & Erro Padrão & Estatística t & P-valor \\
\hline C & 0.787723 & 0.985131 & 0.799612 & 0.4239 \\
DA & -0.014649 & 0.013508 & -1.084466 & 0.2782 \\
Curso & -0.229317 & 0.309140 & -0.741791 & 0.4582 \\
& & McFadden $\mathrm{R}^{2}=0,00897$ & \\
\hline
\end{tabular}

Como os prospectos mais assinalados são B e A, com $74,88 \%$ e $74,54 \%$ das preferências, respectivamente, isso sugere que, no conjunto que relata situações opostas, porém com os mesmos valores monetários em jogo, a influência dos efeitos certeza e reflexo é independente do desempenho acadêmico auferido e do curso frequentado.

O conjunto 3, constituído dos problemas 4 e 13, demonstra uma violação à suposição de que as decisões são tomadas considerando as probabilidades envolvidas, pois os prospectos são equivalentes e apenas apresentados por formas diferentes. Esse efeito é batizado por Kahneman e Tversky (1979) como efeito isolamento. A Tabela 8 expõe os resultados para o terceiro conjunto. 
Tabela 8

Modelo Geral para o Conjunto 3

\begin{tabular}{ccccc}
\hline & \multicolumn{2}{c}{ Variável dependente: RACIONAL } & & \\
Variáveis & Coeficiente & Erro Padrão & Estatística t & P-valor \\
\hline C & -3.670594 & 1.101879 & -3.331212 & 0.0009 \\
DA & 0.057090 & 0.015284 & 3.735317 & 0.0002 \\
Curso & -0.982420 & 0.323876 & -3.033319 & 0.0024 \\
& & McFadden $\mathrm{R}^{2}=0,0712$ & & \\
\hline
\end{tabular}

Todas as variáveis da estimação para o conjunto 3 mostraram-se estatisticamente significativas, conforme demonstra o p-valor inferior a 5\%. O sinal negativo para a dummy curso sugere que os acadêmicos de Administração realizaram escolhas menos racionais que os discentes de Saúde, isto é, os primeiros apresentaram maior susceptibilidade aos vieses cognitivos, afastando-os das escolhas racionais $\mathrm{AA}$ ou $\mathrm{BB}$. Já a variável desempenho acadêmico (DA) registrou coeficiente positivo, com magnitude de 0,057 , indicando que, quanto maior o desempenho acadêmico dos discentes, maior as suas probabilidades de realizarem escolhas racionais, ou seja, de escolherem AA ou BB.

O conjunto 4, composto dos problemas 5 e 6 , visa avaliar o efeito certeza em problemas que não envolvam valores monetários. A Tabela 9 apresenta os resultados da regressão Logit para o conjunto 4.

Tabela 9

Modelo Geral para o Conjunto 4

\begin{tabular}{ccccc}
\hline & \multicolumn{2}{c}{ Variável dependente: RACIONAL } & & \\
Variáveis & Coeficiente & Erro Padrão & Estatística t & P-valor \\
\hline C & -1.359981 & 1.039121 & -1.308781 & 0.1906 \\
DA & 0.032582 & 0.014604 & 2.231084 & 0.0257 \\
Curso & -1.029165 & 0.330665 & -3.112414 & 0.0019 \\
& & & & \\
\hline
\end{tabular}

Apesar da reduzida capacidade explicativa do modelo, as variáveis centrais da estimação mostraram-se, novamente, significantes sob o ponto de vista estatístico. O sinal negativo da dummy curso sugere, assim como na regressão para o conjunto 3, que os acadêmicos de Administração são mais susceptíveis aos vieses cognitivos. A variável desempenho acadêmico (DA) manteve o padrão das estimações anteriores, com coeficiente pequeno e positivo.

O conjunto 5, constituído pelos problemas 7 e 11, ilustra uma situação em que os valores monetários e as probabilidades em jogo são idênticas, entretanto, as questões apresentam cenários opostos de ganhos e perdas. O objetivo também é avaliar o comportamento de aversão ou propensão ao risco em cenários distintos. A Tabela 10 apresenta os resultados da regressão Logit. 
Tabela 10

Modelo Geral para o Conjunto 5

\begin{tabular}{ccccc}
\hline \multicolumn{5}{c}{ Variável dependente: RACIONAL } \\
Variáveis & Coeficiente & Erro Padrão & Estatística t & P-valor \\
\hline C & 2.199362 & 1.019721 & 2.156827 & 0.0310 \\
DA & -0.032157 & 0.013962 & -2.303241 & 0.0213 \\
Curso & 0.204295 & 0.305163 & 0.669463 & 0.5032 \\
& \multicolumn{3}{c}{ McFadden $\mathrm{R}^{2}=0,0202$} \\
\hline
\end{tabular}

A variável desempenho acadêmico mostrou-se estatisticamente significativa, porém o sinal negativo sugere, nessa situação, diferentemente das demais, a existência de uma relação inversa entre a racionalidade das escolhas e o desempenho acadêmico dos discentes.

O conjunto 6, composto pelos problemas 8 e 12, apresenta duas situações opostas, porém com os mesmos valores monetários em jogo, visando à avaliação do efeito reflexo. Os resultados da estimação da Logit para o conjunto 6 são expressos na Tabela 11.

Tabela 11

Modelo Geral para o Conjunto 6

\begin{tabular}{ccccc}
\hline \multicolumn{5}{c}{ Variável dependente: RACIONAL } \\
Variáveis & Coeficiente & Erro Padrão & Estatística t & P-valor \\
\hline C & -3.027496 & 1.060833 & -2.853885 & 0.0043 \\
DA & 0.042644 & 0.014472 & 2.946707 & 0.0032 \\
Curso & -0.102054 & 0.307332 & -0.332066 & 0.7398 \\
& \multicolumn{3}{c}{ McFadden $\mathrm{R}^{2}=0,0356$} \\
\hline
\end{tabular}

A variável DA mostrou-se, de novo, estatisticamente significante, e seu coeficiente maior que zero é indicativo de uma relação direta entre DA e racionalidade de escolhas.

Assim como no conjunto anterior, o conjunto 7, formado pelos problemas 4 e 10, relata cenários de ganhos e perdas. O viés é verificado pelo comportamento de aversão ao risco no domínio dos ganhos e de propensão ao risco no domínio das perdas. A Tabela 12 fornece os resultados da regressão.

Tabela 12

Modelo Geral para o Conjunto 7

\begin{tabular}{ccccc}
\hline \multicolumn{5}{c}{ Variável dependente: RACIONAL } \\
Variáveis & Coeficiente & Erro Padrão & Estatística t & P-valor \\
\hline C & -3.014553 & 1.063963 & -2.833325 & 0.0046 \\
DA & 0.041427 & 0.014478 & 2.861294 & 0.0042 \\
Curso & -0.164296 & 0.305921 & -0.537054 & 0.5912 \\
& \multicolumn{4}{c}{ McFadden $\mathrm{R}^{2}=0,0326$} \\
\hline
\end{tabular}


Na mesma direção das estimações anteriores, a variável desempenho acadêmico (DA) registrou significância estatística, com sinal positivo e a variável curso mostrou-se insignificante.

\section{Resumo dos resultados}

$\mathrm{Na}$ Tabela 13, a coluna Significância Estatística dispõe de duas possíveis respostas, significante (S) ou não significante (NS), tendo como parâmetro o nível de 5\% de significância. Já para a coluna Sinal do Coeficiente, as classificações são positivo (P) ou negativo (N).

A principal variável avaliada pela pesquisa, o desempenho acadêmico (DA), registrou significância estatística em seis dos sete conjuntos estimados, não apresentando relevância estatística somente no conjunto 2. Nas estimações em que o DA foi significante, somente no conjunto 5 o sinal do coeficiente de DA foi negativo, indicando, dessa forma, a possibilidade de existência de uma relação positiva entre o desempenho acadêmico e a racionalidade das escolhas.

Tabela 13

\section{Resumo dos Resultados}

\begin{tabular}{cccc}
\hline Variáveis & Conjuntos & Significância Estatística & Sinal do Coeficiente \\
\hline DA & 1 & $\mathrm{~S}$ & $\mathrm{P}$ \\
& 2 & $\mathrm{NS}$ & $\mathrm{N}$ \\
& 3 & $\mathrm{~S}$ & $\mathrm{P}$ \\
& 4 & $\mathrm{~S}$ & $\mathrm{P}$ \\
& 5 & $\mathrm{~S}$ & $\mathrm{~N}$ \\
& 6 & $\mathrm{~S}$ & $\mathrm{P}$ \\
& 7 & $\mathrm{~S}$ & $\mathrm{P}$ \\
\hline Curso & 1 & $\mathrm{NS}$ & $\mathrm{P}$ \\
& 2 & $\mathrm{NS}$ & $\mathrm{N}$ \\
& 3 & $\mathrm{~S}$ & $\mathrm{~N}$ \\
& 4 & $\mathrm{~S}$ & $\mathrm{~N}$ \\
& 5 & $\mathrm{NS}$ & $\mathrm{P}$ \\
& & $\mathrm{NS}$ & $\mathrm{N}$ \\
& 6 & $\mathrm{NS}$ & $\mathrm{N}$ \\
\hline
\end{tabular}

A variável binária Curso apresenta significância estatística somente em duas das sete estimações efetuadas. Sua irrelevância nas demais regressões pode ser um sinal de que a variável não é importante para explicar a racionalidade das escolhas.

\section{Conclusão}

O objetivo principal deste trabalho foi verificar se o desempenho acadêmico influencia o processo de tomada de decisão dos agentes frente a problemas que envolvam incerteza, ou seja, se à medida que o desempenho acadêmico se eleva, as escolhas se tornam mais racionais e menos susceptíveis aos vieses cognitivos. Além disso, seguindo os estudos de Kahneman e Tversky (1979), busca-se avaliar a tendência à aversão ao risco no domínio dos ganhos e à propensão ao risco no domínio das perdas.

É importante salientar que o critério para a racionalidade das escolhas não seguiu os parâmetros defendidos pela Teoria da Utilidade Esperada, mas sim a coerência com que os respondentes 
manifestavam suas preferências. Tal critério ressalta que realizar escolhas racionais consiste em conferir o mesmo peso a cenários distintos, ou seja, avaliar uniformemente as probabilidades em situações de ganhos e perdas. Assim, os respondentes que indicavam esse padrão de comportamento tiveram suas respostas classificadas como racionais, enquanto as escolhas dos demais foram tidas como irracionais.

Os resultados sugerem que, assim como nos estudos de Kahneman e Tversky (1979), os respondentes da pesquisa são avessos ao risco no domínio dos ganhos e propensos ao risco no domínio das perdas. Tais comportamentos antagônicos refletem a ação de vieses cognitivos, como o efeito certeza no cenário de ganhos e o efeito reflexo em situação de perdas. Em relação à influência do desempenho acadêmico (DA), os resultados sugerem que exista uma relação direta entre desempenho e racionalidade, pois discentes com DA superior realizaram escolhas mais racionais. Outras variáveis, como Idade, Sexo, Ocupação e Curso não apresentaram significância estatística para explicar a racionalidade das escolhas.

\section{Notas}

${ }^{1}$ Veja os livros Buffett, M., \& Clark, D. (2007). O Tao de Warren buffett (I. Korytowski, Trad.). Rio de Janeiro: Sextante. (Obra original publicada em 2006); Graham, B. (2007). O investidor inteligente (4. ed.). Rio de Janeiro: Nova Fronteira; Como ganhar dinheiro na bolsa. (2008, maio 14). Revista ISTO É, Economia e Negócios, (2010).

${ }^{2}$ Refere-se aos cursos de Medicina, Fisioterapia, Enfermagem, Nutrição e Farmácia.

${ }^{3}$ O SAT (Scholastic Assessment Test) é um exame padronizado nos Estados Unidos aplicado a estudantes do $2^{\circ}$ grau (atual Ensino Médio) e o GMAT (Graduate Management Admission Test) é um exame para seleção em cursos de pós-graduação em Administração de Empresas.

${ }^{4}$ A utilização de dois períodos visa minimizar possíveis influências externas e sazonais no desempenho escolar dos acadêmicos, aproximando a sua nota média de seu desempenho acadêmico real.

${ }^{5}$ A pontuação 75 (setenta e cinco) é utilizada, pois, dado que o número de pontos necessários para ser aprovado em qualquer disciplina da faculdade em estudo são 70 (setenta), a margem de 5 (cinco) pontos é concedida.

${ }^{6}$ Refere-se aos cursos de Medicina, Fisioterapia, Enfermagem, Nutrição e Farmácia.

${ }^{7}$ A avaliação dos resultados por sexo (masculino e feminino) com o objetivo de identificar possíveis desvios de conduta entre estes dois grupos distintos foge ao escopo do presente trabalho. Tal análise pode ser encontrada em Araújo, D. R., \& Silva, C. A. T. (2007). Aversão à perda nas decisões de risco. Revista de Educação e Pesquisa em Contabilidade, 1(3), 45-62; Mineto, C. A. L. (2005). Percepção ao risco e efeito disposição: uma análise experimental da teoria dos prospectos (Tese de doutorado). Universidade Federal de Santa Catarina, Florianópolis, SC, Brasil, e Silva, R. F. M., Lagioia, U. C. T., Maciel, C. V., \& Rodrigues, R. N. (2009). Finanças comportamentais: um estudo comparativo utilizando a teoria dos prospectos com os alunos de graduação do curso de ciências contábeis. Revista Brasileira de Gestão de Negócios, 11(33), 383-403.

${ }^{8}$ É uma técnica estatística que visa a predição de uma variável geralmente binária, por meio de variáveis explicativas contínuas e/ou binárias.

${ }^{9}$ A inclusão destas variáveis segue Barber, B., \& Odean, T. (2001). Boys will be boys: gender, overconfidence, and common stock investment. Quarterly Journal of Ecomomics, 116(1), 261-292. doi: 10.1162/003355301556400, que identificam um comportamento mais próximo da racionalidade em mulheres e indivíduos menos jovens.

\section{Referências}

Araújo, D. R., \& Silva, C. A. T. (2007). Aversão à perda nas decisões de risco. Revista de Educação e Pesquisa em Contabilidade, 1(3), 45-62.

Cappellozza, A., \& Sanchez, O. (2011). Análise de decisões sobre uso de tecnologia: um estudo no setor de telefonia móvel fundamentado nos axiomas da economia comportamental. Revista de Administração Contemporânea, 15(6), 1078-1099. Recuperado de http://www.scielo.br/pdf/rac/v15n6/07.pdf. doi: 10.1590/S1415-65552011000600007 
Chevalier, J., \& Ellison, G. (1999). Are some mutual fund managers better than others? Crosssectional patterns in behavior and performance. Journal of Finance, 54(3), 875-899. doi: $10.1111 / 0022-1082.00130$

Cruz, L. F. B., Kimura, H., \& Krauter, E. (2003). Finanças comportamentais: investigação do comportamento decisório dos agentes brasileiros de acordo com a Teoria do Prospecto de Kahneman \& Tversky. Anais da Assembléia do Conselho Latino-Americano das Escolas de Administração (CLADEA), Lima, Peru, 38.

Gottesman, A., \& Morey, M. R. (2006). Manager education and mutual fund performance. Journal of Empirical Finance, 13(2), 145-182. doi: 10.1016/j.jempfin.2005.10.001

Grinblatt, M., Keloharju, M., \& Linnainmaa, J. (2011). IQ and stock market trading. Journal of Finance, 66(6), 2121-2164. doi: 10.1111/j.1540-6261.2011.01701.x

Kahneman, D., \& Tversky A. (1979). Prospect theory: an analysis of decision under risk. Econometrica, 47(2), 263-292. doi: 10.2307/1914185

Kahneman, D., \& Tversky, A. (1981). The framing of decisions and psychology of choice. Science, 211(4481), 453-458. doi: 10.1126/science.7455683

Kimura, H., Basso, L. F. C., \& Krauter, E. (2006). Paradoxos em finanças: teoria moderna versus finanças comportamentais. Revista de Administração de Empresas, 46(1), 41-58. doi: $10.1590 /$ S0034-75902006000100005

Masood, O., \& Piranfar, H. (2010). Determinants of returns and decisions of fund managers: survey evidence from four Turkish banks. Journal of Asset Management, 11(1), 43-54. doi: 10.1057/jam.2009.25

Mendes-da-Silva, W., \& Yu, A. S. O. (2009). Análise empírica do senso de controle: buscando entender o excesso de confiança. Revista de Administração Contemporânea, 13(2), 247-271. Recuperado de http://www.scielo.br/pdf/rac/v13n2/06.pdf. doi: 10.1590/S141565552009000200006

Reed, S. K. (1999). Cognition théories et applications. Paris: De Boeck Ed.

Rogers, P., Securato, J. R., \& Ribeiro, K. (2007). Finanças comportamentais no Brasil: um estudo comparativo. Revista de Economia e Administração, 6(1), 49-68.

Silva, R. F. M., Lagioia, U. C. T., Maciel, C. V., \& Rodrigues, R. N. (2009). Finanças comportamentais: um estudo comparativo utilizando a teoria dos prospectos com os alunos de graduação do curso de ciências contábeis. Revista Brasileira de Gestão de Negócios, 11(33), 383-403. 


\section{APÊNDICE}

\section{Questionário}

\section{PARTE I}

1. Sexo:

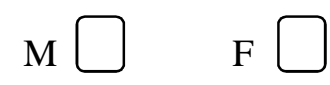

2. Curso:

3. Período:

4. Idade:

5. Ocupação:

\section{PARTE II}

Leia e escolha apenas uma alternativa para cada problema proposto. Não existe resposta correta para os problemas a seguir. A opção escolhida deve representar a sua preferência.

Problema 1: Qual das duas alternativas você prefere?

\section{Alternativa A}

$33 \%$ de chances de ganhar $\$ 2500$

$66 \%$ de chances de ganhar $\$ 2400$

$1 \%$ de chances de ganhar $\$ 0$

Problema 2: Qual das duas alternativas você prefere?

\section{Alternativa A}

$33 \%$ de chances de ganhar $\$ 2500$

$67 \%$ de chances de ganhar $\$ 0$

Problema 3: Qual das duas alternativas você prefere?

\section{Alternativa A}

$80 \%$ de chances de ganhar $\$ 4000$

$20 \%$ de chances de ganhar $\$ 0$

Problema 4: Qual das duas alternativas você prefere?

\section{Alternativa B}

$100 \%$ de chances de ganhar $\$ 2400$

\section{Alternativa B}

$34 \%$ de chances de ganhar $\$ 2400$

$66 \%$ de chances de ganhar $\$ 0$

\section{Alternativa B}

$100 \%$ de chances de ganhar $\$ 3000$ 


\section{Alternativa A}

$20 \%$ de chances de ganhar $\$ 4000$

$80 \%$ de chances de ganhar $\$ 0$

Problema 5: Qual das duas alternativas você prefere?

\section{Alternativa A}

$50 \%$ de chances de ganhar uma viagem de

três semanas para a Inglaterra, França e Itália

$50 \%$ de chances de não ganhar nada

Problema 6: Qual das duas alternativas você prefere?

\section{Alternativa A}

$5 \%$ de chances de ganhar uma viagem de

três semanas para a Inglaterra, França e Itália

95\% de chances de não ganhar nada

Problema 7: Qual das duas alternativas você prefere?

\section{Alternativa A}

$45 \%$ de chances de ganhar $\$ 6000$

$55 \%$ de chances de ganhar $\$ 0$

Problema 8: Qual das duas alternativas você prefere?

\section{Alternativa A}

$0,1 \%$ de chances de ganhar $\$ 6000$

$99,9 \%$ de chances de ganhar $\$ 0$

Problema 9: Qual das duas alternativas você prefere?

\section{Alternativa A}

$80 \%$ de chances de perder $\$ 4000$

$20 \%$ de chances de perder $\$ 0$

\section{Alternativa B}

$25 \%$ de chances de ganhar $\$ 3000$

$75 \%$ de chances de ganhar $\$ 0$

\section{Alternativa B}

$100 \%$ de chances de ganhar uma

viagem de uma semana para a

Inglaterra

\section{Alternativa B}

$10 \%$ de chances de ganhar uma viagem de uma semana para a Inglaterra $90 \%$ de chances de não ganhar nada

\section{Alternativa B}

$90 \%$ de chances de ganhar $\$ 3000$

$10 \%$ de chances de ganhar $\$ 0$

\section{Alternativa B}

$100 \%$ de chances de perder $\$ 3000$ 
Problema 10: Qual das duas alternativas você prefere?

\section{Alternativa A}

$20 \%$ de chances de perder $\$ 4000$

$80 \%$ de chances de perder $\$ 0$

Problema 11: Qual das duas alternativas você prefere?

\section{Alternativa A}

$45 \%$ de chances de perder $\$ 6000$

$55 \%$ de chances de perder $\$ 0$

Problema 12: Qual das duas alternativas você prefere?

\section{Alternativa A}

$0,1 \%$ de chances de perder $\$ 6000$

$99,9 \%$ de chances de perder $\$ 0$

\section{Alternativa B}

$25 \%$ de chances de perder $\$ 3000$

$75 \%$ de chances de perder $\$ 0$

\author{
Alternativa B \\ $90 \%$ de chances de perder $\$ 3000$ \\ $10 \%$ de chances de perder $\$ 0$
}

\section{Problema 13:}

Considere um jogo de dois estágios. No primeiro estágio, existe uma probabilidade de $75 \%$ de que o jogo termine sem que você ganhe nada, e uma probabilidade de $25 \%$ de que se mova ao segundo estágio. Se você atingir o segundo estágio, você pode escolher entre as alternativas a seguir. Observe que a escolha deve ser feita antes do início do jogo.

\section{Alternativa A}

$80 \%$ de chances de ganhar $\$ 4000$

$20 \%$ de chances de ganhar $\$ 0$

\section{Alternativa B}

$100 \%$ de chances de ganhar $\$ 3000$ 